\title{
Management of nicotinamide $N$-methyltransferase overexpression: inhibit the enzyme or reduce nicotinamide intake? Reply to Zhou S, Li D, Zhou Y [letter]
}

\author{
Aimo Kannt ${ }^{1,2}$ - Anja Pfenninger ${ }^{1}$ - Anke Tönjes ${ }^{3} \cdot$ Matthias Blüher $^{3}$
}

Received: 4 June 2015 / Accepted: 8 June 2015 / Published online: 26 June 2015

(C) Springer-Verlag Berlin Heidelberg 2015

Keywords 1-Methylnicotinamide $\cdot$ Adipose tissue $\cdot$ Insulin resistance $\cdot$ Nicotinamide $\cdot$ Nicotinamide

$N$-methyltransferase $\cdot$ NNMT $\cdot$ Type 2 diabetes

\section{Abbreviations \\ MNA 1-Methylnicotinamide \\ NMMT Nicotinamide $N$-methyltransferase}

To the Editor: In their letter to Diabetologia [1], Zhou and colleagues suggest that inhibition of nicotinamide $N$-methyltransferase (NNMT) may not be a viable therapeutic approach for insulin resistance or type 2 diabetes. Instead, the authors propose to reduce nicotinamide intake or increase its elimination, implicating that it is the excess nicotinamide that is causative in the development of metabolic diseases. They postulate that our finding of higher adipose tissue NNMT expression and plasma 1-methylnicotinamide (MNA) levels in patients with insulin resistance and type 2 diabetes [2] may be the result of an induction in NNMT expression by increased nicotinamide intake rather than the metabolic disease itself.

Although we cannot completely rule out that possibility, we think that increased nicotinamide intake is not

Aimo Kannt

aimo.kannt@sanofi.com

1 Sanofi Research and Development, Industriepark Hoechst, H824, D-65926 Frankfurt am Main, Germany

2 Medical Faculty Mannheim, Heidelberg University, Mannheim, Germany

3 Department of Medicine, University of Leipzig, Leipzig, Germany the predominant cause of our previously reported NNMT expression and serum MNA changes or phenotype associations. First, NNMT has been reported to be upregulated in preclinical models of diabetes and obesity, independently of nicotinamide intake [3, 4]. Additionally, adipose tissue NNMT expression was found to be higher in obesity-prone than in obesity-resistant mouse strains [4], and higher urinary MNA concentrations were observed in patients with type 2 diabetes compared with healthy controls, and in $d b / d b$ mice and obese Zucker rats [5], independently of nicotinamide intake. Furthermore, treatment with an antisense oligonucleotide against NNMT was shown to improve insulin sensitivity and to reduce body weight gain in mice fed a high-fat diet compared with mice on the same diet that were treated with vehicle or a non-silencing antisense molecule [4]. Finally, an induction of tissue NNMT expression by nicotinamide has so far only been demonstrated in rats treated with very high doses of nicotinamide [6] exceeding normal dietary intake by several orders of magnitude.

We acknowledge that excess nicotinamide may play a role in the pathogenesis of type 2 diabetes and obesity. Prolonged high niacin or nicotinamide intake has been reported to reduce insulin sensitivity in individuals with normal or impaired glucose tolerance [7-9]. In addition, there is epidemiological evidence demonstrating a chronological link between increased nicotinamide intake and the rising prevalence of obesity and type 2 diabetes [10].

However, it remains unclear whether nicotinamide itself is the culprit in terms of causing metabolic disease and whether this is unrelated to NNMT activity. In fact, there is evidence that the effects of excess nicotinamide on insulin resistance are mediated by MNA, the product of the reaction catalysed by NNMT. First, administration 
of either nicotinamide or MNA to healthy rats had similar effects on glucose homeostasis, resulting in an increase in both blood glucose and insulin concentrations [11]. Second, it was demonstrated that both nicotinamide and MNA led to the generation of reactive oxygen species (ROS) in rats [11, 12] and Caenorhabditis elegans [13] whereas only MNA, not nicotinamide, was effective at triggering oxidative stress in a $C$. elegans mutant devoid of NNMT. Thus, the suggestion of Zhou et al [1] that NNMT inhibition will have similar effects to excess nicotinamide intake is not sufficiently supported by research data, as the latter leads to excessive generation of MNA and ROS, which would be prevented by NNMT inhibition. Additionally, excess nicotinamide and NNMT inhibition have opposite effects on the cellular methylation potential determined by the ratio of S-adenosylmethionine (SAM) to Sadenosylhomocysteine (SAH). Excess nicotinamide has been demonstrated to lower methyl group availability and cause DNA hypomethylation [6] whereas NNMT knockdown led to an increase in the SAM/SAH ratio and histone methylation in vitro [14] and in vivo [4].

In conclusion, we fully agree with Zhou et al that further studies carefully controlling nicotinamide intake and elimination are required to better understand the mechanisms by which nicotinamide metabolism contributes to the pathogenesis of insulin resistance and obesity. However, we do not concur with their suggestion that our observations of increased adipose tissue NNMT expression and plasma MNA levels merely reflect changes in nicotinamide intake or excretion, and that NNMT inhibition and excess nicotinamide would have similar metabolic effects.

Duality of interest AK and AP are employees of Sanofi. Both other authors declare that there is no duality of interest associated with this manuscript.

Contribution statement $\mathrm{AK}$ drafted the manuscript. AP, AT and MB revised it for important intellectual content. All authors approved the final version.

\section{References}

1. Zhou SS, Li D, Zhou Y (2015) Management of nicotinamide Nmethyltransferase overexpression: inhibit the enzyme or reduce nicotinamide intake? Diabetologia doi:10.1007/s00125-0151-3677-6

2. Kannt A, Pfenninger A, Teichert L et al (2015) Association of nicotinamide- $N$-methyltransferase mRNA expression in human adipose tissue and the plasma concentration of its product, 1-methylnicotinamide, with insulin resistance. Diabetologia 58:799-808

3. Riederer M, Erwa W, Zimmermann R, Frank S, Zechner R (2009) Adipose tissue as a source of nicotinamide $N$-methyltransferase and homocysteine. Atherosclerosis 204:412-417

4. Kraus D, Yang Q, Kong D et al (2014) Nicotinamide $\mathrm{N}$-methyltransferase knockdown protects against dietinduced obesity. Nature 508:258-264

5. Salek RM, Maguire ML, Bentley E et al (2007) A metabolomic comparison of urinary changes in type 2 diabetes in mouse, rat, and human. Physiol Genomics 29:99-108

6. Li D, Tian YJ, Guo J, Sun WP et al (2013) Nicotinamide supplementation induces detrimental metabolic and epigenetic changes in developing rats. Br J Nutr 110:2156-2164

7. Chang AM, Smith MJ, Galecki AT, Bloem CJ, Halter JB (2006) Impaired beta-cell function in human aging: response to nicotinic acid-induced insulin resistance. J Clin Endocrinol Metab 91:3303-3309

8. Kahn SE, Beard JC, Schwartz MW et al (1989) Increased beta-cell secretory capacity as mechanism for islet adaptation to nicotinic acid-induced insulin resistance. Diabetes 38:562-568

9. Greenbaum CJ, Kahn SE, Palmer JP (1996) Nicotinamide's effects on glucose metabolism in subjects at risk for IDDM. Diabetes 45:1631-1634

10. Zhou SS, Li D, Zhou YM, Sun WP, Liu QG (2010) B-vitamin consumption and the prevalence of diabetes and obesity among the US adults: population based ecological study. BMC Public Health 10:746

11. Zhou SS, Li D, Sun WP et al (2009) Nicotinamide overload may play a role in the development of type-2 diabetes. World J Gastroenterol 15:5674-5684

12. Li D, Sun WP, Zhou YM et al (2010) Chronic niacin overload may be involved in the increased prevalence of obesity in US children. World J Gastroenterol 16:2378-2387

13. Schmeisser K, Mansfeld J, Kuhlow D et al (2013) Role of sirtuins in lifespan regulation is linked to methylation of nicotinamide. Nat Chem Biol 9:693-700

14. Ulanovskaya OA, Zuhl AM, Cravatt BF (2013) NNMT promotes epigenetic remodeling in cancer by creating a metabolic methylation sink. Nat Chem Biol 9:300-306 